Volume 9, No.1.3, 2020

International Journal of Advanced Trends in Computer Science and Engineering

Available Online at http://www.warse.org/IJATCSE/static/pdf/file/ijatcse7491.32020.pdf

https://doi.org/10.30534/ijatcse/2020/7491.32020

\title{
SIE-EVD: A Novel Satellite Image Enhancement Technique with Quality Metrices for Effective Visual Display using CBIR
}

\author{
Neetu Manocha ${ }^{1}$, Rajeev Gupta ${ }^{2}$ \\ ${ }^{1}$ M.M. Institute of Computer Technology \& Business Management (MCA) \\ neetu0019@gmail.com \\ 2 Department of Computer Science and Engineering, MMEC \\ rajeev.gupta@mmumullana.org \\ Maharishi Markandeshwar (Deemed to be University), Mullana, \\ Ambala, Haryana, India
}

\begin{abstract}
With the remarkable development and usage of remote sensing, an extra-ordinary level of visual quality required in the captured satellite images. Satellite image enhancement and quality maintenance are some of the most challenging tasks for the researchers in the field of digital image processing. Due to environmental conditions or inbuilt camera capabilities, a satellite capture and produce an image having blur or other types of noises. The captured images consist of lots of information about the surface of the earth or other planets. But, due to blur or other types of noise, the quality of these images is fallen. Eminent researchers were already proposed several conventional image enhancement techniques to increase the quality of a satellite image. But, after the detailed research, the authors have analyzed that most of the existing techniques do not produce an accurate result. In this context, a new satellite image enhancement technique must be developed for effective visual display. This paper presents a detailed discussion of the proposed satellite image enhancement framework (SIE-EVD) to reduce the blur or noise of an image without losing high-frequency details for the effective visual display by using CBIR. The proposed framework is tested on the dataset of Bhuvan - a national Geo-portal developed and hosted by ISRO (Indian Space Research Organisation). The performance of the proposed framework is evaluated by using accuracy and response time.
\end{abstract}

Key words: Image enhancement, Satellite imagery, Image noise, Color, Blur, Brightness, Sharpness, De-noise, Histogram effect, Histogram graph

\section{INTRODUCTION}

In the present digital society, the remote sensing field shows tremendous growth towards the digital era and has set an important position in the day-to-day life of every person. Satellite imagery data captured by a good quality in-built camera fixed in satellites with supportive technology stacks play a vital role in all many image processing applications which include Oceanography, Bio-diversity conservation, Agriculture and Farming, Landscaping, Fishing, Meteorology, Forestry, Cartography, Intelligence, Regional planning, Military and Security, Education, Weather forecasting, Geology, etc. [1]. This captured satellite imagery contains very sensitive or confidential information related to the surface of the earth or other planets. Due to environment messiness and incompatible setting or dealing of camera used in satellite, a satellite image contains blur or other types of noises [2], which degrades the quality of images

Noise in a digital image is a random variation in brightness or color information of an image. Various types of noises affect satellite imagery. These noises include dark current noise, quantization noise, Shot noise, Speckle noise and Amplifier noise [3]. The main reasons behind such type of noises in satellite imagery are:

$\checkmark$ Disturbance in Environment,

$\checkmark$ Zooming quality of a focal lens,

$\checkmark$ Continuous motion of Satellite while capturing the image,

$\checkmark$ Distance from the target object, and

Spreaded Optical deformation.

It is very important to enhance these degraded images for a better outcome. Figure 1 shows some blur images captured by satellites.

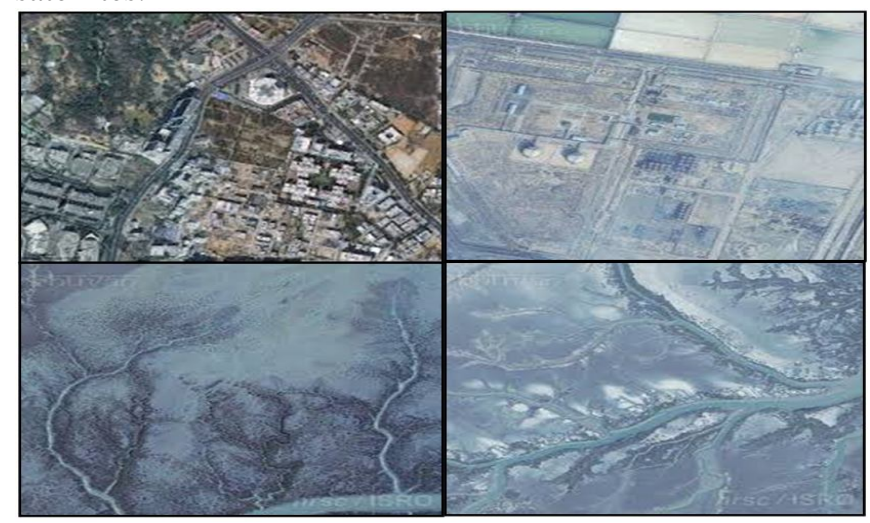

Figure 1: Blurry Satellite Images (source: www.bhuvan.nrsc.gov.in) 
A solution named SIE-EVD (Satellite Image Enhancement for Effective Visual Display) is proposed that will deal with blurred satellite imagery capturing on less constrained conditions and non-ideal environments. To simulate experiments a tool named OpenCV-Python is used. OpenCV-Python is the Python API of OpenCV. It combines the best qualities of OpenCV C++ API and Python language. It supports a lot of algorithms related to Computer Vision and Machine Learning. Paper is organized as follows: - Section-2 covers some related prior work about satellite image enhancement in recent years. Section-3 describes the proposed solution. Section-4 describes the experimental results and performance analysis of the proposed framework. At last, Section-5 concludes about overall efforts made in the formulation of the research and future directions.

\section{RELATED WORK TOWARDS SATELLITE IMAGE ENHANCEMENT}

Various eminent researchers were already worked on the enhancement of Satellite imagery in past years. Table 1 highlights some related prior work about satellite image enhancement in recent years.

Table 1: Related work towards Satellite Image Enhancement

\begin{tabular}{|c|c|}
\hline Authors/Year & Description \\
\hline Tarik et al./2009 & $\begin{array}{c}\text { Authors [4] proposed a new framework that was based on histogram } \\
\text { equalization. The proposed framework was resolved an optimization } \\
\text { problem and also reduce the cost. }\end{array}$ \\
\hline $\begin{array}{l}\text { Hasan et al./2010 } \\
\quad \& 2011\end{array}$ & $\begin{array}{l}\text { Authors [5][7] suggested a new satellite imagery enhancement } \\
\text { method. The proposed method was based on the computation of the } \\
\text { high-frequency of the images. The proposed method show the better } \\
\text { outcome in terms of PSNR and Root-mean-square-error as } \\
\text { comparison to conventional methods [7]. }\end{array}$ \\
\hline Bhandari et al./2011 & $\begin{array}{l}\text { Researchers [6] proposed a singular value decomposition (SVD) and } \\
\text { discrete cosine transform (DCT) based contrast enhancement } \\
\text { technique for contrast enhancement of low-contrast satellite images. }\end{array}$ \\
\hline Neena et al./2012 & $\begin{array}{l}\text { Authors [8] proposed an image enhancement technique with the } \\
\text { concern of resolution and contrast. The proposed technique followed } \\
\text { the bi-cubic interpolation, DWT, SWT and SVD. This technique has } \\
\text { shown very good results over conventional methods, but it required a } \\
\text { high computational cost. }\end{array}$ \\
\hline Randa et al./2013 & $\begin{array}{l}\text { Authors [9] presented a DCT and SVD based satellite image contrast } \\
\text { enhancement method. The result of the proposed method shows better } \\
\text { than conventional methods. }\end{array}$ \\
\hline Virender et al./2016 & $\begin{array}{l}\text { Researchers }[10] \text { reviewed the semantic-based image retrieval } \\
\text { techniques from cyberspace for further image enhancement. }\end{array}$ \\
\hline N. E.O. Ofek/2019 & $\begin{array}{l}\text { Authors [11] suggested a software tool for solving the astrometry } \\
\text { problem of correctly matching of astronomical images. }\end{array}$ \\
\hline Muhammad et al./2019 & $\begin{array}{l}\text { Researchers [12] proposed a methodology to detect buildings from } \\
\text { low-contrast satellite images. The proposed method achieves high } \\
\text { performance thus return more heterogenous and perceptive results } \\
\text { over conventional techniques. }\end{array}$ \\
\hline Gautam et al./2019 & $\begin{array}{l}\text { Researchers [13] analyzed that Norman AI retrieved Inkblot images } \\
\text { from the concerned dataset for performing the further psychological } \\
\text { interpretation concern test. }\end{array}$ \\
\hline
\end{tabular}

\section{SIE-EVD: SATELLITE IMAGE ENHANCEMENT FOR EFFECTIVE VISUAL DISPLAY}

\subsection{Algorithm of Proposed Framework}

The algorithm of the proposed framework SIE-EVD is as follows:

Input: Satellite Image (with blur or any other noise)

Output: Enhanced Satellite Image

Var image, image_size, blur_value, rb_img, dnoise_img, sm_img

Begin
Step-1: image $=$ Select the image from dataset Apply Step-2 to 5, to get the enhanced image

Step-2: Calculate Blur of selected image image_size $=$ Compute Image-Size

blur_value = variance_of_laplacian(image)

Display image_size, blur_value

Step-3: Remove Blur from selected image rb_img = 2D_filter_threshold (image)

Step-4: Denoise the resultant image dnoise_img = FastN1MeansDenoisingColored (rb_img)

Step-5: Add smoothness in the resultant image

sm_img = GaussianBlur (dnoise_img)

Go to Step-9

Apply Step- 6, 7 and 8 (if required)

Step-6: Draw Histogram Graph and apply Histagram Effect

Step-7: Apply Color Effects on resultant image

Step-8: Apply Sharp, Contrast and Brightness effects

Step-9: Display resultant image End

\subsection{Block diagram of proposed framework}

The block diagram of the proposed framework is shown in figure 2. Here, an image having noise or blur is taken from the database of Satellite Images captured by Satellite(s). After that, blurriness within the image will be calculated and further enhancement operations will perform. Finally, an enhanced satellite image will be retrieved from the system.

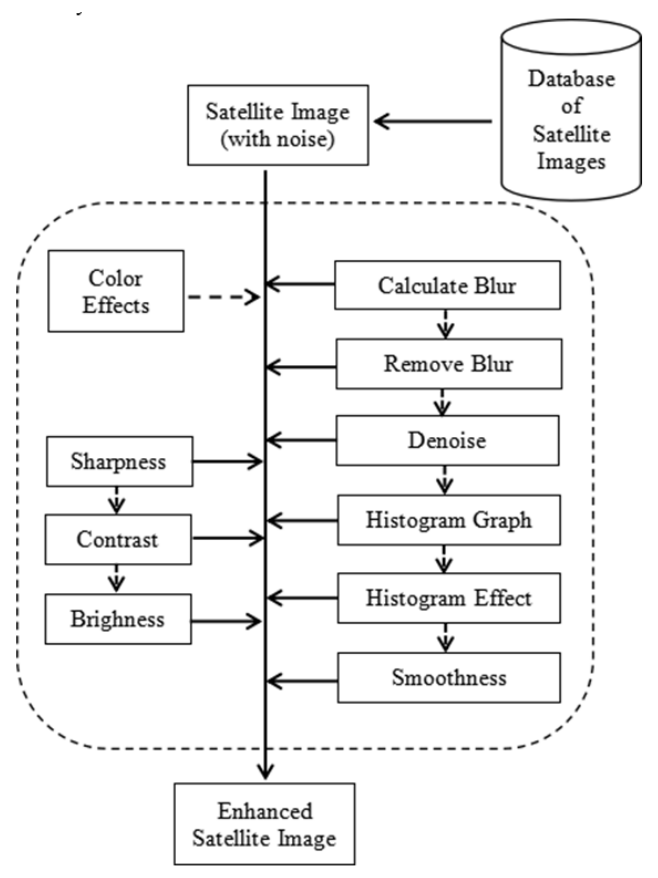

Figure 2: Block Diagram of SIE-EVD 


\section{EXPERIMENTAL RESULTS AND PERFORMANCE ANALYSIS}

\subsection{Dataset}

For the experimental analysis, many datasets consisting of satellite images are available. These datasets are available either paid or free. Bhuvan is a national Geo-portal. It is developed and hosted by ISRO (Indian Space Research Organization). The purpose of this portal is to manage Geo-spatial data, Tools for research analysis and related services. It is available free to registered researchers. Bhuvan portal has many features like a) free downloading of satellite images, b) Satellite Imagery's data representation in Visual form, c) Clustering and analysis of data, and d) downloading the reports, etc. The Satellite imageries can be visualized in the form of 2D and 3D, which are Multi-platform, multi-temporal and Multi-sensor in nature. It supports many real-life applications, which are governed by various departments of Central and State Government, Research Institutes, and public as well private industries.

\subsection{Sample images used for experimentation}

The experimentation, implementation, and testing were done based on the sample images fetched from Bhuvan-a national Geo-portal developed and hosted by ISRO (Indian Space Research Organisation) dataset. The following images shown in Table 2 were used for experimentation.

Table 2: Sample Images with basic properties for experimentation

(source: www.bhuvan.nrsc.gov.in)

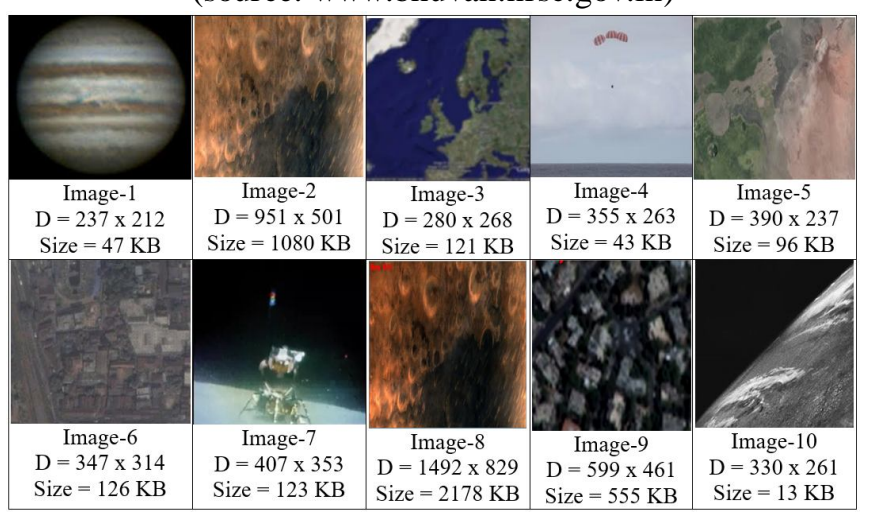

$* \mathrm{D}=$ Dimension

\subsection{Screenshots of proposed framework}

The following figures demonstrate the working of the proposed Satellite Image Enhancement for Effective Visual Display (SIE-EVD).

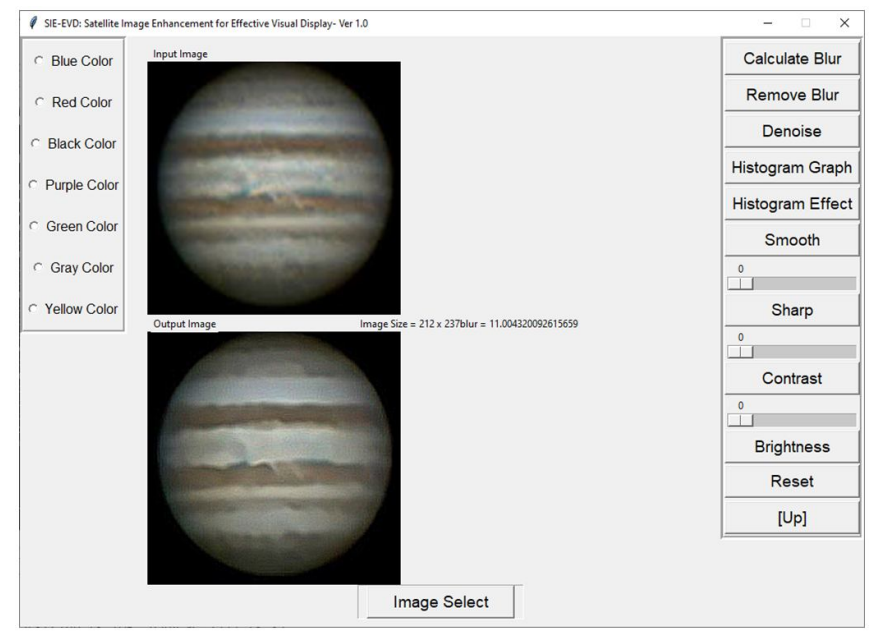

Figure 3: Denoise and Remove Blur effects on selected image

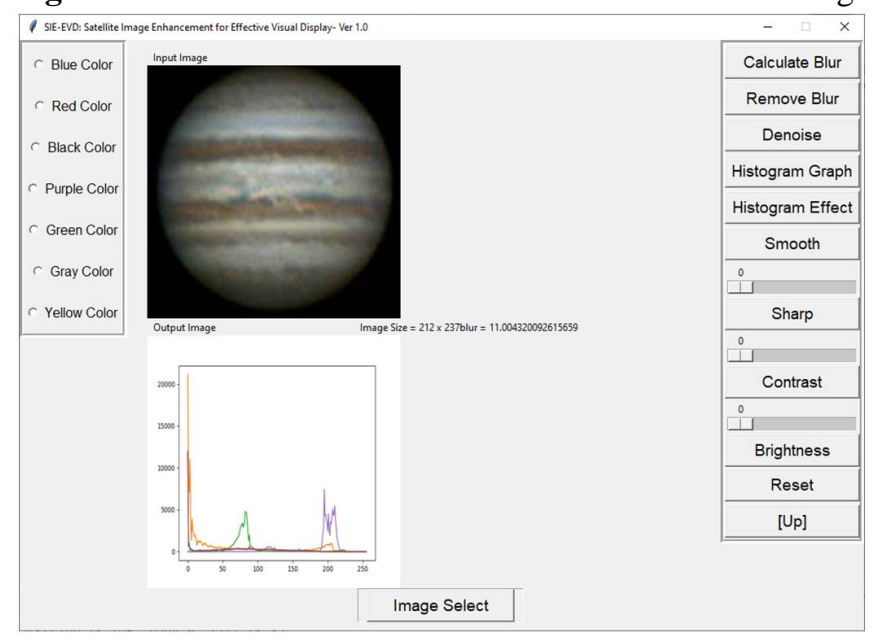

Figure 4: Histogram Graph of selected image

The various color effects on selected image in SIE-EVD is shown in the figure 5

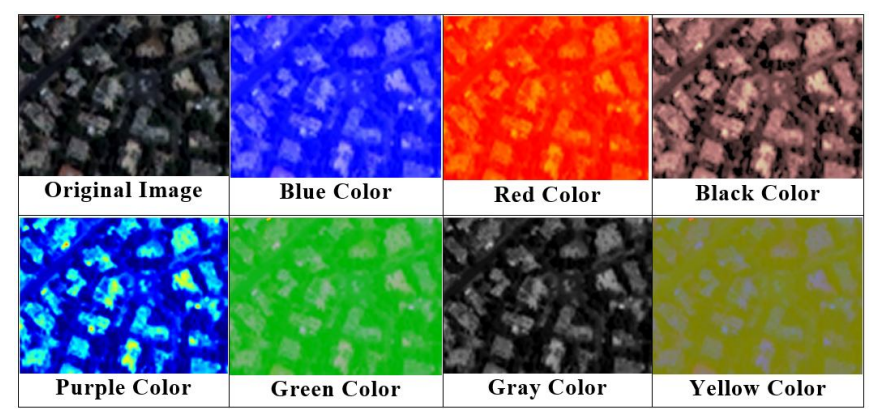

Figure 5 : Color effects on selected image

\subsection{Performance Analysis of proposed framework with existing techniques:}

The Table 3 shows the blur value of sample images, computed value and relevant accuracy (in percentage) of CLAHE, Bi-Lateral Filter, Power Law Transformation as well as SIE-EVD (proposed) techniques. 
Table 3: Computed Value and relevant accuracy (in \%) of sample images

\begin{tabular}{|c|c|c|c|c|c|c|c|c|c|}
\hline \multirow[b]{2}{*}{$\begin{array}{l}\text { Blurry } \\
\text { Images }\end{array}$} & \multirow[b]{2}{*}{ 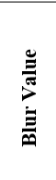 } & \multicolumn{2}{|c|}{ CLAHE } & \multicolumn{2}{|c|}{ Bi-Lateral Filter } & \multicolumn{2}{|c|}{$\begin{array}{c}\text { Power Law } \\
\text { Transformation }\end{array}$} & \multicolumn{2}{|c|}{$\begin{array}{l}\text { SIE-EVD } \\
\text { (Proposed) }\end{array}$} \\
\hline & & 总 & 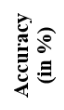 & 离 & 预 & 总 & 总。 & 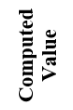 & $\begin{array}{l}\mathrm{n} \\
\mathrm{U}\end{array}$ \\
\hline Image-1 & 11.00 & 717.74 & 92.82 & 715.19 & 92.85 & 646.39 & 93.54 & 327.61 & 96.72 \\
\hline Image-2 & 13.10 & 858.34 & 91.42 & 4187.89 & 58.12 & 130.34 & 98.70 & 128.64 & 98.71 \\
\hline Image-3 & 14.22 & 690.58 & 93.09 & 1223.52 & 87.77 & 422.37 & 95.78 & 230.41 & 97.70 \\
\hline Image-4 & 14.94 & 1021.52 & 89.78 & 1218.9 & 87.81 & 166.9 & 98.33 & 159.46 & 98.41 \\
\hline Image-5 & 28.64 & 829.9 & 91.70 & 1405.87 & 85.94 & 404.86 & 95.95 & 403.85 & 95.96 \\
\hline Image-6 & 31.73 & 573.33 & 94.27 & 2199.57 & 78.00 & 584.25 & 94.16 & 502.07 & 94.98 \\
\hline Image-7 & 37.56 & 541.33 & 94.59 & 797.34 & 92.03 & 907.9 & 90.92 & 289.91 & 97.10 \\
\hline Image-8 & 97.07 & 497.07 & 95.03 & 854.73 & 91.45 & 546.29 & 94.54 & 64.96 & 99.35 \\
\hline Image-9 & 61.86 & 378.49 & 96.22 & 1243.9 & 87.56 & 543.52 & 94.57 & 67.01 & 99.33 \\
\hline Image-10 & 96.00 & 636.91 & 93.63 & 1176.88 & 88.23 & 435.66 & 95.64 & 428.44 & 95.72 \\
\hline
\end{tabular}

Figure 6 shows the graphical representation of experimental performance analysis with the concern of relevant accuracy (in percentage) of sample images by CLAHE, Bi-Lateral Filter, Power Law Transformation as well as SIE-EVD (proposed) techniques.

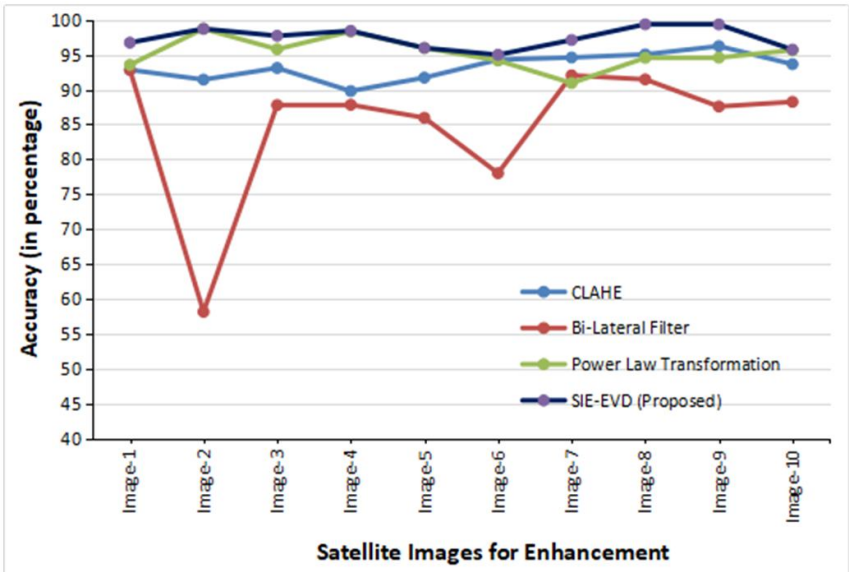

Figure 6 : Enhancement Accuracy (in percentage) of Sample Images

\section{CONCLUSION}

In this paper, a new framework SIE-EVD is proposed for further enhancement of Satellite images. In performance analysis, the proposed algorithm is compared with existing CLAHE, Bi-Lateral Filter and Power Law Transformation techniques. After the experimentation, the proposed algorithm performance is better than conventional satellite image enhancement techniques. As per results received, on-an-average, SIE-EVD shows approximately $4 \%$ improvement as compare to CLAHE, approximately $13 \%$ improvement as compare to Bi-Lateral Filter and approximately $2 \%$ improvement as compare to Power Law Transformation techniques

\section{ACKNOWLEDGEMENT}

Authors acknowledges support and inputs from family members, colleagues, and other supporting staff of Maharishi Markandeshwar (Deemed to be University), Mullana, (Ambala), India, for giving the new motivation about this field. Authors also acknowledges and thankful to Bhuvan (bhuvan.nrsc.gov.in) for use of satellite images.

\section{REFERENCES}

1. N. Manocha and R. Gupta, "A Comparative Analysis of Existing Satellite Image Enhancement Techniques for Effective Visual Display", Journal of Computational and Theoretical Nanoscience, vol. 16, no. 9, (2019), pp. 4003-4007. https://doi.org/10.1166/jctn.2019.8285

2. R. Gupta and A. Kumar, "Noisy iris recognition and its importance", Journal of Ultra Scientist of Physical Sciences, vol. 25, no. 2B, (2013), pp. 229-234.

3. M. Zhang and B. Gunturk, "A new image denoising method based on the bilateral filter", IEEE International Conference on Acoustics, Speech and Signal Processing, Las Vegas, NV, (2008), pp. 929-932.

4. T. Arici, S. Dikbas and Y. Altunbasak, "A Histogram Modification Framework and Its Application for Image Contrast Enhancement", in IEEE Transactions on Image Processing, vol. 18, no. 9, (2009), pp. 1921-1935. https://doi.org/10.1109/TIP.2009.2021548

5. H. Demirel and G. Anbarjafari, "Satellite Image Resolution Enhancement Using Complex Wavelet Transform",in IEEE Geoscience and Remote Sensing Letters, vol. 7, no. 1, (2010), pp. 123-126.

6. A. K. Bhandari, A. Kumar and P.K. Padhy, "Enhancement of low contrast satellite images using discrete cosine transform and singular value decomposition", World Academy of Science, Engineering and Technology, vol. 55, (2011), pp. 35-41.

7. H. Demirel and G. Anbarjafari, "Discrete Wavelet Transform-Based Satellite Image Resolution Enhancement," in IEEE Transactions on Geoscience and Remote Sensing, vol. 49, no. 6, (2011), pp. 1997-2004. https://doi.org/10.1109/TGRS.2010.2100401

8. K.A. Neena, A. Raj and R. C. Roy, "Image Enhancement based on Stationary Wavelet Transform, Integer Wavelet Transform and Singular Value Decomposition", International Journal of Computer Applications, vol. 58, no. $11,(2012)$, pp. 30-35.

9. R. Atta and M. Ghanbari, "Low-contrast satellite images enhancement using discrete cosine transform pyramid and singular value decomposition", IET Image Processing, vol. 7, no. 5, (2013), pp. 472-483. 
10. V. Singh and R. Gupta, "Semantics Based Image Retrieval from Cyberspace - A Review Study", International Journal of Advanced Research in Computer Science, vol. 7, no. 4, (2016), pp. 16-21.

11. N.E.O. Ofek, "ACode for Robust Astrometic Solution of Astronomical Images", The Atronomical Society of the Pacific, vol. 131, no. 999, (2019)

https://doi.org/10.1088/1538-3873/ab04df

12. Muhammad Aamir, Yi-Fei Pu, Ziaur Rahman, Muhammad Tahir, Hamad Naeem, and Qiang Dai, "A Framework for Automatic Building Detection from Low-Contrast Satellite Images", Symmetry, vol. 11, no. 1, (2019), pp. 3.

13. G. Kumar, G. Singh, V. Bhatanagar and and K. Jyoti, "Scary dark side of artifificial intelligence: A perilous contrivance to mankind", Humanities \& Social Sciences Reviews, vol. 7, no. 5, (2019), pp.1097-1103.

https://doi.org/10.18510/hssr.2019.75146 